Dassisti M., Panetto H., Tursi A. (2006). Product-driven Enterprise Interoperability for Manufacturing Systems Integration. Proceedings of the BPM2006 Business Process Management Workshops. $2^{\text {nd }}$ ENEI Workshop, Vienna, Austria, September 4, 2006. Springer Verlag, Lecture Notes in Computer Science, September, LNCS 4103, 249-260, ISBN 3-540-32595-6

\title{
Product-driven Enterprise Interoperability for Manufacturing Systems Integration
}

\author{
Michele Dassisti ${ }^{1}$, Hervé Panetto ${ }^{2}$, Angela Tursi ${ }^{1}$ \\ ${ }^{1}$ Dipartimento di Ingegneria Meccanica e Gestionale, Politecnico di Bari, Italy \\ m.dassisti@poliba.it, a.tursi@poliba.it \\ ${ }^{2}$ Centre de Recherche en Automatique de Nancy (CRAN - UMR 7039), \\ Nancy-University, CNRS, France \\ Herve.Panetto@cran.uhp-nancy.fr
}

\begin{abstract}
The "Babel tower effect", induced by the heterogeneity of applications available in the operation of enterprises brings to a consistent lack of "exchangeability" and risk of semantic loss whenever cooperation has to take place within the same enterprise. Generally speaking, this kind of problem falls within the umbrella of interoperability between local reference information models .This position paper discuss some idea on this field and traces a research roadmap to make enterprise interoperable on the basis of this statement to face interoperability of RIMs by focusing the attention on the product. By applying a transformation between local information models into an "ontological reference model” centred on the product it is possible to insure applications interoperability.
\end{abstract}

Keywords: Interoperability, Integration, Product Data Management, Enterprise Model, Ontology.

\section{Introduction}

There are many tools available which cause information overload in the operation of enterprises for several scopes: managerial, production, marketing and so on. This information is characterised by a common problem: the "Babel tower effect" induced by the heterogeneity of applications (such as ERP, SCM, PDM, MES ...), of users and of domains. All this information is strongly influenced mainly by two drivers: the scope (say, criteria) for which this has been collected and used, and the subjectivity induced by each decision maker using it (say interpretation or classification of information).

This situation is due to different information reference models embedded into applications, and thus into a lack of "exchangeability" and a loss of semantics whenever a different use of information is required. This latter kind of problem falls within the umbrella of interoperability problems where, generally speaking, interoperability can be defined as that intrinsic characteristic of a generic entity (organization, system, process, model, ...) allowing its interaction with other entities - 
to a different extent of simplicity - to cooperate for achieving a common goal (second level goal) within a definite interval of time, while pursing its own specific goal (first level goal).

In principle, local reference information models (RIM) embed many classes of information, which responds to specific information classification criteria. Criteria thus define "dimensions", and according to these it is possible to retrieve information whenever required: the heterogeneity of information comes from the specific nature of this classification depending on the two referred drivers. Whenever it is possible to translate these dimensions into, say, neutral dimensions, it would be possible to reach easily a true interoperability between RIMs.

This concept is here translated in practice by referring these RIMs to the common element in all manufacturing operations: the product. The product, which evolves through time (in a diachronic way) along its life cycle, allows thus to define a common reference information model, to support information exchange between the product views and the many applications that refer to them.

The basic assumption of the thesis here sustained is that it is possible in principle to transform local information models (embedded into local applications) to an “ontological reference model” centred on the product (PCORM). In order to insure applications interoperability, the PCORM should be embedded into the product itself; that means information should be deeply linked to the product and possibly recorded into it to be retrieved by applications whenever required.

This position paper presents some idea and traces a research roadmap on this topic, to explore the possibility to make enterprise interoperable on the basis of productcentred information view. The shape of the paper is as follows: section 2 introduces standards and frameworks concerned on information interoperability; section 3 provides the conceptual idea of the paper; section 4 presents some hints for the research roadmap; section 5 summarises the research challenges that have to be faced in the next future and finally in section 6 some general comments are provided.

\section{Information Interoperability}

Enterprises need to focus on their core-competences to improve efficiencies. At the present, the product is perceived again, after the soap-bubble new-economy experiences, the real value of enterprises and a new role is emerging for the whole production process.. In this scenario, the product and production lifecycle and its related management are unavoidably turning to be key aspects, defining a sort of "product centric" or "product-driven" reference point-of-view [1]. The integrated management of all the information regarding the "product" and its manufacturing is one of the related questions. Information interoperability asks for common shared approaches: standardisation and enterprise modelling methodologies are the most advanced tools to this aim. 


\subsection{Standards for Product data representation and exchange}

Standardisation initiatives interesting for our purpose are the IEC 62264 set of standards [3] and the ISO 10303 Technical specifications [4], because they are related to Product Data Management at the business and the manufacturing levels of enterprises.

The IEC 62264 set of standards specify a set of reference models for information exchange to facilitate the integration of business applications and manufacturing control applications, within an enterprise. The key aspects for integrating the business applications and the manufacturing operations and control applications are the information structures and exchanges, related to the products, managed by activities, applications, processes, resources, and functions.

Examples of enterprise applications dealing with these exchanges are, at the business levels, ERP (Enterprise Resource Planning) systems or PDM (Product Data Management) systems and, at the manufacturing level, MES (Manufacturing Execution Systems), to name only a few. In particular, MES functions relate production monitoring including materials (raw and finished) and resources (equipment and personnel) traceability information. The International Organization for Standardization (ISO) has been pushing forward the development of standards and models to foster the exchange of information related to goods and services [5]. Efforts like ISO 10303 STEP - Standard for the Exchange of Product model data - deals with the issues of integration and interoperability problem. STEP represents the standard for the computer-interpretable representation of product information and for the exchange of product data. It aims to provide a neutral mechanism capable of describing products throughout their lifecycle. Nowadays, STEP has been recognized as appropriate to help in the integration of manufacturing systems in industries such as automotive, aircraft, shipbuilding, furniture, building and construction, gas and oil.

A significant solution for PDM data exchange is the Unified PDM Schema, which is a basic specification for the exchange of administrative product definition data. It has been created by unifying all PDM data between all existing STEP Application Protocols, such as AP-203, AP-214 and AP-227 and allows the exchange of information that is stored in PDM systems. This information typically forms the metadata for any product. In order to deal with the increasing demands on product models exchange, the standard has specified a set of STEP reusable modules related to PDM. These modules concern all related information attached or describing products technical data such as product structure, configuration control, efficacy, person and organisations, etc. Data integration ensures that the information describing product design, manufacturing and life cycle support is defined only once; STEP data integration eliminates redundancy and the problems caused by redundant information.

\subsection{Enterprise Modelling Methodologies}

Several enterprise modelling methodologies and supporting tools, addressing phases of the enterprise life cycle and various aspects of enterprise modelling, have been developed so far. These methodologies and tools are intended to support business decision-making (such as process visualisation and simulation), enterprise 
process management, control and monitoring of operational processes (such as workflow) and performance monitoring (such as visualisation of work in progress). Some of the most relevant methodologies and tools are here briefly recalled, highlighting main features and criticalities for the scope of this position paper.

The ZACHMAN's framework [6] is a logical structure for classifying and organising the descriptive representations of an Enterprise. The framework bundle of information consists in representing the design artefacts that constitute the intersection between the roles in the design process It results in a balance between the holistic view and the pragmatic view, in a sort of a framework that results a good classification scheme more oriented to problem solving and planning tasks.

GERAM [7, 8] framework encompasses all knowledge needed for enterprise engineering / integration. The completeness of the architecture, which encompasses architectures, methodologies, languages, modelling concepts, models, tools, operation systems for Enterprise Modelling makes this approach a reference for the ISO 15704 standard [20], i.e. an useful guidelines and a way to create a common ontology in the scientific community, more than a specific tool to be used for interoperability.

GRAI [9, 10] methodology proposes a modelling framework to support the analysis, design and implementation of an enterprise, referring to two dimensions, based on the systemic approach: functional abstraction and decomposition levels. GIM modelling framework, which is included into the GRAI methodology, introduces the decision dimension/view which is not taken into account in other modelling frameworks: in this sense, it is more decision support oriented.

ARIS [11, 12] approach is based on the "multiple views" concept: the objective is to reduce the complexity by dividing the enterprise into individual views, by referring to a number of modelling languages co-ordinated into the Control View. The ARIS architecture forms the framework for the development and optimisation of integrated information systems as well as a description of their implementation by superimposing a structure of interrelations; it is also based on a process-oriented approach [10].

CIMOSA [13] is a framework to analyse the requirements of enterprises and translating these into a system which enables and integrates the functions that match the requirements. CIMOSA is neutral in this regard: it addresses concepts and models that are only necessary to model integrated enterprise systems, focusing on process model based enterprise activity control. There is no explicit consideration on interoperability issues in CIMOSA modelling framework. However, CIMOSA can be a contribution for integrated paradigm to establish interoperability (see ISO 14258 [21] and ENV 40003 [22]).

DoDAF [14] is one of the most comprehensive enterprise frameworks and provides a good understanding of the stakeholders and users needs. It provides poor contribution to integrated platforms, model-driven design and generation of interoperable solutions. Most all leading Enterprise Architecture vendors are supporting DoDAF, such as METIS Enterprise from Troux Tecnology, System Architect from Popkin, Mega Suite form Mega International, and the other top ten providers.

TEAF (Treasury Enterprise Architecture Framework) [14] is another of the most comprehensive enterprise frameworks and gives a good understanding of the 
stakeholders and users and the minimum of information required to align business and IT. The contribution to integrated platforms, model-driven design and generation of interoperable solutions is poor.

The AKM [15] technology and corresponding methodology is based on the concepts of Enterprise Knowledge Spaces, and its four knowledge dimensions:: Approach - Methodology -Infrastructure and Solutions - AMIS. The AKM technology is prone to interoperability, since it implements the layered Enterprise Architecture, and it is prepared for the POPS methodology, for the Enterprise Knowledge Architecture and the Intelligent Infrastructure services.

ISO 15745 - Open System Application Integration Framework [23] consists of the application integration framework (AIF), which defines generic elements and rules for describing integration models and application interoperability profiles, together with their component profiles (process profiles, information exchange profiles, and resource profiles). It supports the development of interface specifications in the form of application interoperability profiles (AIPs) enabling the selection of suitable resources and the documentation of the "as built" application. A key factor is the concept of interfaces: the interfaces of the resources used to perform the function are configured to work with the corresponding resource interfaces of the other functions, involved in a target manufacturing applications. There is no complete industrial implementation reported so far; furthermore, this approach can work only if various standards used to specify interfaces are interoperable between them.

MISSION [16] approach extends the High Level Architecture (HLA) approach $[17,18,19]$ includes the modelling aspects which describe how to collect the necessary data for the distributed simulation, specification on how the different simulation models can be coordinated starting from a template library; a simulation manager supports the definition and the interoperability of simulation templates by exchanging objects. Even this interesting solution relies on a top-down specification of objects to be exchanged and the related interfacing information in the federation.

Table 1 summarises the standards and enterprise modelling approaches for interoperability discussed above. Till now, no unified conceptual basis for modelbased enterprise engineering -enabling consistency, convergence and interoperability of the various modelling methodologies and supporting tools - has been created so far based on a bottom-up view.

From this brief summary it is evident how all the solutions available so far share the same common top-down approach, .relying on the process-view approach, which belongs to the static and holistic perception of the enterprise, descending from system theory. To summarise, it is evident how a different conception for the global information reference model is required for cooperation to take place effectively; this justifies the thesis here sustained that the product view may turn to be very interesting, since the product is in some sense "inertial" with respect to the changing cooperation processes and the potential dynamical evolution in time of actors. 
Table 1. Standards and frameworks comparison.

\begin{tabular}{llc}
\multicolumn{1}{c}{$\begin{array}{c}\text { Standard/ } \\
\text { Framework }\end{array}$} & \multicolumn{1}{c}{ Interoperability core features } & $\begin{array}{c}\text { Product } \\
\text { view }\end{array}$ \\
\hline IEC 62264 & Information structures and exchanges & Yes \\
\hline ISO 10303 & $\begin{array}{l}\text { Representation of product information } \\
\text { and exchange of product data }\end{array}$ & Yes \\
\hline ZACHMAN & $\begin{array}{l}\text { Intersection between roles in design } \\
\text { process and product abstractions }\end{array}$ & No \\
\hline GERAM & $\begin{array}{l}\text { Components used in all types of } \\
\text { enterprise integration processes }\end{array}$ & No \\
\hline GRAI & $\begin{array}{l}\text { Modelling with reference to functional } \\
\text { abstraction and decomposition levels }\end{array}$ & No \\
\hline ARIS & $\begin{array}{l}\text { Development and optimisation of } \\
\text { integrated information systems }\end{array}$ & No \\
\hline CIMOSA & $\begin{array}{l}\text { Concepts and models strictly necessary to } \\
\text { model integrated enterprise systems }\end{array}$ & No \\
\hline DoDAF & $\begin{array}{l}\text { Understanding of the stakeholders and } \\
\text { users needs }\end{array}$ & No \\
\hline TEAF & $\begin{array}{l}\text { Information from stakeholders and users } \\
\text { alignment in business and IT }\end{array}$ & No \\
\hline AKM & $\begin{array}{l}\text { Layered Enterprise Architecture, POPS } \\
\text { methodology, Enterprise Knowledge and } \\
\text { Intelligent Infrastructure services }\end{array}$ & No \\
\hline ISO 15745 & $\begin{array}{l}\text { Interfaces } \\
\text { Distributed discrete-event simulation }\end{array}$ & No \\
\hline
\end{tabular}

\section{Product View for Reference Information Models}

It has to be stated firstly that each application adopted for the operation of enterprises uses its own information repository, which serves for its own scopes. Each repository refers to a reference information model (RIM) that specifies the structure of the information treated and its nature. This model embeds several design and management criteria related to the scope of the application to which it is devoted (say subjective part), even though a information is still valid independently of the application itself (say, objective part).

It is thus possible to state that each application retrieves information from its repositories, according to the specific need during its operation: the efficiency of this "information retrieval process (IRP)" is directly dependent on the amount of objective information available. A negative effect results from the subjective part of information, in case this has to be imported from different applications: in this latter case, the translation required might bring to strong loss of information, and thus it may have impact on its significance or pertinence. 
Let us imagine that a reference system can be defined for making explicit information concerning the transformations the product has to undergo. This information is always a result of a decisional process made by operators according to their specific scope (top-down approach): in this case the information reference system is the operator himself (see $i_{\mathrm{RS}}{ }^{1,2,3, \ldots}$ in Fig. 1 ). The novelty of our idea is to reverse the information reference system (see $i_{\mathrm{RS}}{ }^{*}$ in Fig. 1 ) by simply making the "product" active part of its transformation process (bottom-up approach).

By referring to the "product view" here proposed, it is possible to guarantee a sort of neutrality of the information, thus reducing the risk of subjectivity and consequently the efficiency of the information retrieval process itself.

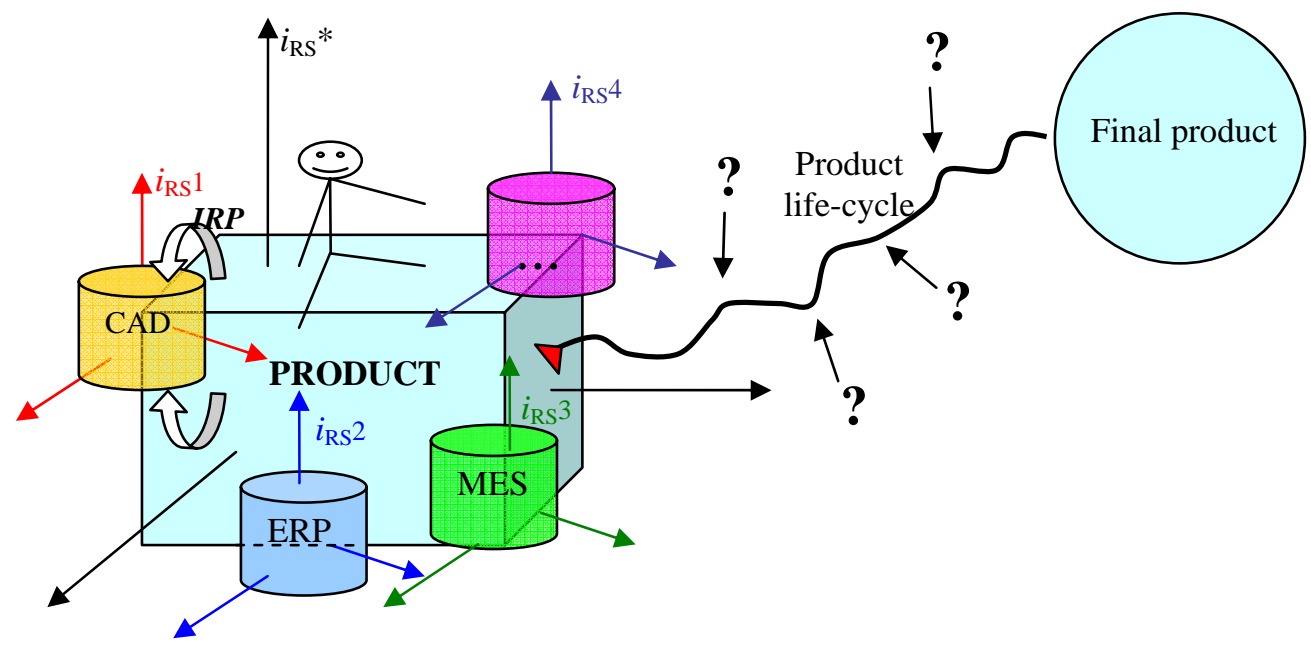

Fig. 1. Product view representation: $\boldsymbol{i}_{\mathbf{R S}}{ }^{*}$ is the "inertial" reference system centred on product

The efforts devoted to information standardisation, as stated before, is a proof of the need to improve the efficiency of IRP, whether its constraints are in the "topdown" approach, which unavoidably limits its significance to the decision-maker capability of capturing all the relevant aspects of RIM. The product-view approach can be defined, on the contrary, as a "bottom-up" one, where adaptively the product "asks" for information to proceed along its life-cycle (see Fig. 2). This representation is obviously not complete, since features and behavioural rules of the "product" are always designed a-priori by the decision maker: it will be a matter of the future research effort to devise if and how it will be possible to allow a pure adaptive mechanism of the class "product", in order to implement a true bottom-up fashion if ever possible.

In order to explain better the product view and the related research challenges, let us imagine a man to "sit on the product" and walk with it during its life-cycle: all the information required to know where to go, which operation to undergo or what kind of information to receive for the next change of state, will turn to have a radically 
different meaning - and consequently structure - with respect to the classical topdown approach.

Starting from this representation, the first step of the research effort in the development of the product-driven interoperability will be the study of the existing reference information models used by the actual applications concerned with the product life-cycle, to find an unique "product view" representation, that will be the basis to design the structure of the PCORM. For the sake of simplification, one can state that the PCORM will result a meta-model derived from a sort of intersection (or any similar reasonable logical operation on models) of the entire specific RIM corresponding to each of the application available in the factory, independently of their objective or subjective nature.

It will be another question of the future research to understand the nature and the contents of this logical operation onto the RIMs (or the repositories of information themselves, in case of instantiation in a real application).

\section{Research on Transformation of Reference Information Models}

The concept of interoperability has been widely analyzed, in the last decade, by the research European community (see IDEAS Roadmap, UEML [24], INTEROP NoE [25] and ATHENA IP [26] projects), in a joint effort between industrial world, tertiary industry, suppliers and academic world. On the basis of results reached so far, the fundamental aspects that can be further explored in the development of the interoperability concept are:

$\checkmark$ the development of synergies between different domains, such as the enterprise modelling, the ontology building and the software architectures and platforms;

$\checkmark$ the definition and the realisation of methods and tools providing an effective support to the collaboration between experts in the various domains.

A step of the research on product-driven interoperability will be to study the RIMs, embedded into the local applications, such as PDM systems and ERP systems. These applications deal with the product but with different points of view; this has to be done by focusing on the product, tracing its diachronic evolution through time along its life cycle.

Starting from this, the research has to define the transformation operations of local reference information models (RIMs) into an "ontological reference model" centred on the product (PCORM). The idea of this position paper could be inspired to the concept of "schema integration", developed in the Database Management System (DBMS) domain, where various data models (namely, schemas) and architectures are used to design the conceptual structure of databases, which is to design an abstract, global, understandable view of the application.

Batini et al [27] define schema integration as "the activity of integrating the schema of existing or proposed databases into a global, unified schema". The contexts in which this concept can be applied are:

$\checkmark$ the view integration (in database design), which produces a global conceptual description of a proposed database, 
$\checkmark$ the database integration (in distributed database management), which produces the global schema of a collection of database.

In both contexts, the approach consists in designing an integrated schema (i.e. a conceptual view in the case of view integration and a global schema in the case of database integration), starting from the single component schema to be integrated (namely: the user views in the view integration and the local schema in the database integration), with the scope of capturing the meaning of data.

In a similar way, to obtain the "ontological reference model" before mentioned it will be necessary to build an integrated schema, containing all the information included in local applications RIMs as necessary (corresponding to the component schemas in the DBMS domain). This "ontological reference model” will be built upon the product, which is the guideline for the design process and to implement information, thus resulting in the bottom up approach.

The framework provided in [27] to face the schema diversity can be a good guideline to suggest a set of steps of the integration process, useful to transform local RIMs into PCORM. Inheriting from the DBMS domain, the integration process can be performed by referring to an ordered mixture of the following activities:

$\checkmark$ pre-integration: it consists of an analysis of local applications RIMs before integration to decide upon some integration policy, what applications to integrate, the order of integration and all additional information relevant to integration;

$\checkmark$ comparison of the schemas: this activity has the scope to determine the correspondences among elements of RIMs and detect possible syntactic or semantics conflicts. Conflicts may rise for several reasons: different perspectives (each application has its own way of modelling the same information), equivalence among constructs of the model (several combinations of constructs can have the same meaning), incompatible design specifications (erroneous choices in inputs to the models integration process). In case common elements may be modelled in different ways, an inter-schema properties (i.e. semantic relationships holding between different models) need thus to be recognised;

$\checkmark$ conforming the schemas: an effort is required to solve conflicts, depending on their nature, to make possible merging of various local RIMs;

$\checkmark \quad$ merging and restructuring: once conflicts are eliminated, the RIMs can be superimposed to derive the intermediate "ontological reference model" with an iterative process: at the end of this, the final "ontological reference model" can be derived. It will require a test against the following qualitative criteria:

o completeness and correctness: the ontological reference model must contain all concepts present in any RIM correctly;

o minimality: the concept represented in more than one RIM must be represented only once in the ontological reference model;

o understandability: the ontological reference model should be easy to understand by the designers.

All of the above activities can be performed for building the PCORM, bearing in mind that all the integration activities are strongly influenced by the data model 
adopted to build the conceptual schemas. As a consequence, these activities might be used only as guidelines or hints for the transformation process of RIMs into the PCORM. Other approaches can be feasible to this aim.

In [28], for example, the integration between PDMs and workflow management systems (which are two integration systems) is suggested using the PDM to store sets of definitions of both the parts and the task that need to be executed on the parts. The PDM acts as the reference database both for the enactment services of the production workflow system and for other systems and manages the Product Breakdown Structure (PBS), the Assembly Breakdown Structure (ABS) and the Work Breakdown Structure (WBS).

In [29], on the other hand, an approach how to link "incompatible" integration systems is suggested, which can represent a valid indication to the research project in exam. The authors introduce a four-layered architecture, which is only a conceptual solution for integration: two partners with the need for co-operation define structural and behavioural patterns of interaction.

All the interaction solutions discussed above are not suitable as a general integration solution for every kind of co-operation between the two or more partners; these can represent a good hint for our research roadmap to devise the transformation operations of local reference information model into the "ontological reference model" centred on the product. Hopefully, an appropriate mix of these can be a good choice to the scope posed for the research.

\section{$5 \quad$ Roadmap of Research}

Following the premises developed in paragraph 3 and 4, herewith we summarise some of the most important research challenges to be faced in the next future for supporting enterprise product-driven interoperability.

The research activities will be centred on the information modelling for the technical management of products and its use for interoperability of the manufacturing applications. These research activities will tend to build on the concept of interoperability based on product view, to be used diffusely in manufacturing cycle, starting from the ideation phase up to the product use.

The methodologies to be used may refer to the design mechanisms of computer science engineering - such as UML standard and formal logic - for the definition of model structures of information within the enterprise. These can ensure a common logical structure for the interoperability of the applications, by referring to the product.

In modelling the various phases of product lifecycle, all the sets of technical information need to be considered, constantly interpreted from the point of view of product; information produced during the idea and design phase need to be also considered. The information model based on the product will constitute a sort of "backbone" for tracing the product evolution and to build the ontological model, making easier the information exchange between different applications linked to the product. 
The final expected outcome of the research plan here described will be the formalization of a common information model, almost indifferent to the application type used in manufacturing systems and based on existing standards, such as ISO 10303 and IEC 62264 previously recalled.

Main focus points of the research will be:

i. study of information reference systems and of applications main models and standard, for the information management of the product;

ii. development of a general reference model, starting from a specific application of PDM (Windchill) and an one for ERP (Sage Adonix), based on a real case of an Italian factory which manufactures bicycles, for the generation of a reference ontology of product information;

iii. formal check of reference model for the ontology of product information, applicatory check on an instanced industrial case for the operational feasibility.

\section{Conclusions}

The interoperability is a concept of growing interest for the enterprise, urged from the competitive pressure imposed by the new market without boundaries. The product is the key concept shared by all applications inside an enterprise: it may serve as a kind of "mediator" to refer all information produced and used by these applications. The thesis here sustained is that an ontological reference model centred on the product takes into account all product points of view.

It is evident how the temporariness of the cooperation is a critical factor for the realization of collaborative networks between manufacturing enterprises, in order to survive in the global market. The scientific studies proposed various answers to this problem: many of these answers can be classified as top-down. The use of a reference system as proposed, in a bottom-up fashion, promises good results in the research of design solution, effective for the temporary cooperation between systems of enterprises, which today has not been reached yet.

Acknowledgments. This work is partially supported by the Commission of the European Communities under the sixth framework programme (INTEROP Network of Excellence, Contract N 508011, <http://www.interop-noe.org>).

\section{References}

1. Morel G., Panetto H., Zaremba M.B., Mayer F.: Manufacturing Enterprise Control and Management System Engineering: paradigms and open issues. IFAC Annual Reviews in Control. 27/2, 199-209, Elsevier, December 2003, ISSN: 1367-5788 
2. Terzi S., Cassina J., and Panetto H.: Development of a metamodel to foster interoperability along the product lifecycle traceability. Proceedings of the IFIP/ACM SIGAPP INTEROPESA conference, Interoperability of Enterprise Software and Applications, February 23-25, Geneva, Switzerland, Springer Science publisher, pp. 1-11, (2005) ISBN: 1-84628-151-2

3. IEC 62264: Enterprise-control system integration, Part 1. Models and terminology, Part 2: Model object attributes. ISO/IEC, (2002) Geneva

4. ISO/TS 10303: STEP modules related to Product Data Management. Industrial automation systems and integration - Product data representation and exchange (2004) Geneva

5. International Organization for Standardization (ISO). Geneva (2005) www.iso.org

6. http://www.zifa.com

7. Bernus P. and Nemes L.: Requirements of the Generic Enterprise Reference Architecture and Methodology. Annual Reviews in Control, 21 (1997) 125-136

8. Bernus P. and Nemes L.: A framework to define a generic enterprise reference architecture and methodology. Computer Integrated Manufacturing Systems, 9 (1996) 179-191

9. Chen, D., Vallespir, B. and Doumeingts, G. : GRAI integrated methodology and its mapping onto generic enterprise reference architecture and methodology. Computers in Industry, 33 (1997) 387- 394

10.McCarthy, I. and Menicou M.: A classification schema of manufacturing decisions for the GRAI enterprise modelling technique. Computers in Industry, 47 (2002) 339-355

11.Scheer A.-W. et al.: ARIS, Business Process Framework, 3 rd edition, Berlin (1999)

12.http://www.pera.net/Methodologies/ARIS/ARIS.html

13.http://www.cimosa.de

14.http://www.gcn.com/enterprisearchitecture

15.http://www.akmii.net

16. Mertins K., Rabe M., Jäkel F-W.: Distributed modelling and simulation of supply chains. International Journal of Computer Integrated Manufacturing, 18/5:342-349, 2005

17.Dahmann J., Fujimoto R. and Weatherly R: The DoD High Level Architecture: an update. (1998)

18.Department of Defence: Defence modelling and simulation Office: High Level Architecture Run-time Infrastructure. Programmer's Guide Version 4 (1998)

19.Zhuge H., Chen J., Feng Y., and Shi Y.: A federation-agent-workflow simulation framework for virtual organisation development. Information \& Management, 39 (2002) 325-336

20.ISO IS 15704: Industrial automation systems - Requirements for enterprise reference architectures and methodologies (1999) Geneva

21.ISO IS 14258: Industrial automation systems - Concepts and rules for enterprise models (1999) Geneva

22.CEN ENV 40003:1990 - CIM Systems Architecture - Framework for Enterprise Modelling

23.ISO/DIS 15745-1: Industrial automation systems and integration - Open systems application integration frameworks - Part 1: Generic reference description; (2000), Geneva

24.http://www.ueml.org

25.http://www.interop-noe.org

26.http://www.athena-ip.org

27.Batini C., Lenzerini M., and Navathe S.B.: A Comparative Analysis of Methodologies for Database Schema Integration. ACM Computing Surveys, 18(4) (1986) 323-364

28.Kovacs Z., Le Goff J.M. and McClatchey R.: Support for product data from design to production. Computer Integrated Manufacturing Systems, 11(4) (1998) 285-290

29.Karcher A. and Glander M.: Global distributed engineering - integrating different process paradigms. Journal of Materials Processing Technology, 138 (2003) 31-137 